\title{
Que peuvent les marchés ?
}

\author{
Philippe CHALMIN \\ Professeur d'Economie — Paris Dauphine \\ $<$ Philippe.Chalmin@dauphine.fr>
}

\begin{abstract}
What can do the markets? This article examines through the light of current and recent past the contributions and limits of agricultural futures markets. Despite the crisis of agricultural raw materials, the markets seem to have very well played their role. If developed countries must stay under the discipline of markets, despite their tendancy to "irrational exuberance" one must recognize that markets do not ensure the development of poor countries. So, it is necessary to allow those countries to deploy autonomous agricultural policies, which means that they can protect themselves from fluctuations in external markets.
\end{abstract}

Key words: agricultural future market, speculation, anticipation, least developed countries

\section{Deux mutations}

Que peuvent les marchés? Quel degré d'efficience convient-il de leur accorder, étant entendu qu'un examen s'impose et qu'on ne peut plus désormais les suivre les yeux fermés et adhérer à ce qui a été un dogme durant tant d'années, à savoir que les marchés ont toujours raison.

Cette question très large, avant de tenter de l'éclairer en gardant le cap sur les marchés agricoles, je souhaiterais l'aborder par l'intermédiaire de deux remarques préalables.

\section{En Europe: \\ une liberté des prix recouvrée}

Pour moi le fait marquant, le principal événement survenu en Europe ces dernières années, ne porte pas sur les anticipations concernant l'après 2013, ni même sur les évolutions des relations entre agriculture, industrie et grande distribution. Pour moi, cet événement n'est pas moins que cette révolution, survenue dans le silence le plus absolu et par laquelle depuis l'été 2006, la Commission sans dire mot, a abandonné toute forme de gestion des marchés céréaliers.

Une parenthèse ouverte le 15 août 1936, lorsque I'Assemblée Nationale vota la loi de création de l'Office du blé, s'est ainsi refermée pour la France, mettant un terme pour les céréales - le grand produit agricole symbole -à soixante-dix ans d'un régime administré. Depuis l'été 2006 nous vivons désormais en Europe dans une sorte de non-PAC, une sorte d'entre-deux, dans lequel il existe encore des règles - un prix $d$ 'intervention et pour le mettre en œuvre des taxes à l'exportation - mais qui ne sont plus appliquées et pour la première fois depuis 1936, les prix européens des céréales sont totalement corrélés au marché mondial, ce qui, vous en conviendrez, représente une véritable révolution culturelle qui explique $d^{\prime}$ ailleurs les émois d'un certain nombre d'agriculteurs soudain confrontés à une nouveauté pour leur métier, celle de se retrouver face à un prix instable et d'être ainsi obligé d'anticiper, d'être ainsi obligé, au sens étymologique du terme, de spéculer.

Je pense que lorsque l'on rédigera à nouveau quelques histoires de l'agriculture européenne, à la fin du $X X^{e}$ et au début du $X X I^{e}$ siècle, plus que la flambée des prix, plus que la crise des marchés que nous venons de connaître en 2007-2008, c'est bien ce moment très important pour la PAC qui marque l'abandon de facto par Bruxelles, de son rôle de stabilisateur, de gardien de la stabilité des prix pour le producteur, qui sera retenu (même si le prix d'intervention existe toujours et que probablement les restitutions seraient rétablies si les prix s'effondraient). Une date qui marque également l'abandon de toute forme de stabilisation pour les consommateurs et c'est ainsi qu'on a pu voir les prix du blé filer aux hauteurs que $I^{\prime}$ on sait, et atteindre près de $300 \$$ la tonne sur le marché à terme de Chicago.

\section{Pays du Sud les moins avancés: les excès de la dérégulation}

Le deuxième point que je souhaiterais mettre en avant - et je rejoindrais ici les propos de Marcel Mazoyer - renvoie aux excès de la dérégulation qui a été imposée ces quinze-vingt dernières années aux pays du Sud où l'on a vu (et fait) tomber les unes après les autres les politiques agricoles héritées de la colonisation, les offices, les « caistab » (caisses de stabilisation), les boards, qui n'étaient pas forcément toujours exemplaires mais qui avaient bien souvent le mérite d'isoler le petit agriculteur des fluctuations des marchés internationaux. La responsabilité des organismes de Washington (Banque mondiale, FMI), avec leurs programmes d'ajustements structurels est grande mais aussi celle des séquelles et des conséquences de nos propres politiques agricoles qui ont provoqué conjointement l'effondrement que I'on sait des marchés à la fin du $X X^{\mathrm{e}}$ siècle, ruinant tout espoir, pour nombre de pays du Tiers-monde, de pouvoir un jour mettre en place des politiques leur assurant une autosuffisance alimentaire, lesquelles ne pouvaient que leur apparaître rationnellement et financièrement dénuées de sens.

Parler de l'efficience du marché aujourd'hui oblige ainsi à se situer entre ces deux mutations qu'ont connu les marchés européens et les marchés des pays du Tiers-monde.

Arrivé à ce point permettez-moi d'entreprendre un éloge du marché.

\section{Éloge du marché}

\section{Une spéculation qui ne date pas d'hier}

Je vous rappellerai tout d'abord que tout ce qui nous a été révélé aujourd'hui des marchés dérivés et des marchés financiers - ils viennent de faire preuve de tant d'excès qu'il semble difficile de les défendre - finalement ne nous apprend pas grand-chose car tout a commencé sur les marchés agricoles. C'est dans ce secteur, souvenez-vous, que tout a été inventé. Relisez ici les nombreux ouvrages et articles sur la Tulipominia, cette spéculation folle que connut le marché des bulbes de tulipes à Amsterdam entre 1637 et 1640 et vous y verrez décrits les premiers marchés de gré à gré, les premiers swaps, les premières options même 
si cela n'est pas formalisé de cette manière. Le premier marché à terme officiel, organisé avec une caisse de compensations qui ait existé sur la planète, a été créé au Japon pour le riz et fonctionna grossièrement entre 1700 et 1850 dans la plaine du Kansai. Et la mère de tous les marchés, le premier marché à terme de l'ère moderne, la base de tous les marchés dérivés de la planète aujourd'hui, fut mise en place vers 1840, à Chicago, sur le marché des œufs (à une époque où les poules pondaient encore de manière saisonnière). C'est sur le modèle de Chicago, confronté à l'émergence de l'instabilité monétaire et financière, que se sont développés les «financial futures ${ }^{1}$ ». Pour la petite histoire, le premier "swap ${ }^{2}$ " fut un swap sucrier en 1980, réalisé en particulier par une maison de négoce française qui eut son heure de gloire, "Sucres et denrées », avec I'organisme d'État qui vendait le sucre des Philippines.

Il est vrai que lorsque l'on considère le développement des marchés dérivés financiers, quand on voit les "errements » auxquels nous ont mené l'inventivité débridée de ceux que l'on appelle les « quants », les financiers quantitatifs, ceux qui rêvent de faire le master de Nicole El Karaoui à I'École Polytechnique, à Paris VII ou le 203 à Dauphine, il est vrai que l'on peut douter du bien-fondé d'une défense de ce genre de marché. Je voudrais simplement attirer votre attention sur la différence qu'il y avait et qu'il y a toujours aujourd'hui entre le développement des dérives financiers effectué pratiquement sans contrôle, sans aucune transparence, sans aucun mécanisme international de surveillance, marchés qui sont devenus des réseaux électroniques mondiaux, et qui sont purement de papier, pour lesquels la notion même de relation à des fondamentaux physiques n'existe plus et nos marchés de matières premières de produits agricoles. Sur ces marchés également il est nécessaire qu'ily ait des échanges de papier, il est nécessaire qu'il y ait de la spéculation, il n'en reste pas moins qu'à la dernière heure du dernier jour de l'échéance finale, le physique doit être livré : il y a donc un moment où se rétablit et s'impose dans toute sa rigueur le lien étroit entre le marché du physique et le marché du papier. Ceci est le premier point que j'entendais souligner.

\section{Des marchés agricoles représentatifs}

Le deuxième point qu'il faut bien garder à l'esprit est le fait que pour l'instant encore, un cer-

\footnotetext{
${ }^{1}$ Contrat à terme

${ }^{2}$ Swap = produit dérivé financier objet d'un contrat d'échange de flux financiers entre deux contreparties, qui sont généralement des banques ou des institutions financières [NDLR]
}

tain nombre de grands produits agricoles relèvent d'un cadre presque classique de marché organisé, tel le marché de Chicago ou le Matif pour Euronext. Ce sont des marchés où l'enregistrement réglementaire des transactions conduit à une transparence à peu près totale des opérations, et où s'exerce de plus le contrôle d'autorités professionnelles de marché ainsi que le contrôle d'autorités nationales $\left(\mathrm{I}^{\prime} \mathrm{AMF}^{3}\right.$ pour la France, la CFTC ${ }^{4}$ aux ÉtatsUnis), en quelque sorte les gardiens et les garants de l'« honnêteté » des transactions. II faut souligner la très grande différence entre les marchés organisés selon ces règles - ceux du maïs, du blé, du soja, du café, du cacao, du sucre - et les marchés pour lesquels il $n^{\prime} y$ a que du physique et pas de marchés représentatifs, comme ceux du riz, des produits laitiers, des viandes, etc. sur lesquels il peut y avoir de temps en temps le développement de marché de gré à gré $\left(\mathrm{OTC}^{5}\right)$ mais ce n'est pas suffisant. Les principes y sont les mêmes mais sans la transparence des marchés organisés.

\section{Un effet limité de la spéculation}

Bien sûr il est clair que le fonctionnement des marchés en général fait appel à un ressort (et à un mot) qui nous a toujours extraordinairement gêné dans le contexte français - le seul point, sur lequel dans le village de notre enfance, l'instituteur et le curé se retrouvaient unis dans leur jugement pour honnir et vouer aux gémonies ses adorateurs - je veux parler bien sûr de l'argent. II est clair que par principe les marchés tels qu'ils fonctionnent font appel à la spéculation. Pour tenter de désarmer la bombe morale que représente ce mot, je rappellerai que la spéculation devient un geste naturel dès l'instant où l'environnement au sein duquel nous sommes plongés se révèle instable. Si Marcel Mazoyer était amené à effectuer une mission aux États-Unis dans les trois mois qui viennent, s'imposerait aussitôt à lui la nécessité de décider du moment auquel il achètera ses dollars. À partir du moment où les cours sont fluctuants, et compte tenu des frais de mission généreusement alloués par l'administration centrale, cette décision ne sera pas sans conséquence pour la qualité de son séjour. Et ce choix d'un jour, d'ici la date de son départ, sera par essence spéculatif. C'est l'instabilité monétaire qui conduit à spéculer, à la hausse ou à la baisse, à réaliser l'achat au plus vite, ou à le différer. Un tel comportement n'avait pas de raison d'être dans l'environnement monétaire fixé par les accords de Bretton Woods, et ce, jusqu'en 1971. Mais le monde est désor-

\footnotetext{
${ }^{3}$ Autorité des marchés financiers

${ }^{4}$ Commodities Futures Trading Commission

${ }^{5}$ Over The Counter en anglais (hors Bourse)
}

mais marqué au coin de l'instabilité, et il nous faut donc nécessairement anticiper sur ce que sera le prix de demain, prix qui sera lui-même le résultat de la somme des anticipations de l'ensemble des acteurs sur ce que sera demain le rapport offre/demande. Nous sommes définitivement entrés dans un univers de spéculation. II faut comprendre que ce n'est pas la spéculation qui crée l'instabilité. J'utilise toujours cette image de l'écume sur la vague : lorsque la mer est plate, lorsque le marché est parfaitement stabilisé par des organismes publics ou autres, il n'y a pas d'écume (de spéculation) ou très peu. Si le vent se lève, sous l'effet d'un déséquilibre offre/demande, la mer se creuse, et avec les vagues la mer blanchit, et l'on ne peut plus faire la différence entre l'écume et l'eau (des fondamentaux). C'est exactement cela le rôle de la spéculation.

J'ajouterais que les comportements spéculatifs, peuvent apparaître qu'il y ait ou non marché dérivé. Le blé et le riz par exemple cette année ont connu des flambées comparables alors que ces deux produits sont totalement différents du point de vue des marchés. À Chicago et à Rouen, le blé est l'objet de marché dérivés, avec spéculation financière, le riz relevant quant à lui d'un marché classique (physique), plus ou moins contrôlé par un cartel de négociants chinois à Bangkok, ce qui ne l'a pas empêché de passer de 250 \$à un peu plus de $1000 \$$ la tonne quand sur la même période, le boisseau $(27 \mathrm{~kg})$ de blé, augmentait également d'un facteur 4 et passait de 2,50 \$à $13 \$$.

Toutes les études qui ont cherché à mesurer l'impact de la spéculation (la « mauvaise » spéculation : celle des « horribles » financiers aux doits griffus se nourrissant de la substance des bons producteurs et des gentils consommateurs) ont ramené son impact à peu près à rien. Je vous renvoie aux travaux de Woking un économiste américain, effectués dans les années 1950 sur les marchés à terme agricoles aux États-Unis et les marchés non ferreux à Londres. Il a montré, en comparant le fonctionnement des marchés des pommes de terre en Californie avant et après la mise en place du marché à terme, combien ce dernier du fait de sa fonction d'anticipation jouait un rôle de stabilisateur. Plus près de nous, la délégation du Trésor du ministère des Finances a publié cet été une étude (qui doit se trouver sur le site de Bercy) sur l'impact de la spéculation financière sur le comportement des marchés agricoles et là encore la conclusion est à peu près la même. Aux États-Unis, certains membres du Congrès ayant eu l'intention de demander la suppression des marchés à terme, le Congrès a diligenté une étude à la Commodity Futures Trading Commission qui ne portait pas sur les produits agricoles, mais sur l'impact de la spéculation 
sur le prix du pétrole à terme, étude qui est arrivée là encore à la même conclusion.

Je ne suis pas certain que l'on doive pour autant accorder à la spéculation et à la fonction de la spéculation la totalité du crédit qu'elles souhaiteraient se voir attribuer du fait qu'elles apporteraient une autostabilisation des anticipations des opérateurs. Car il n'est pas rare que la volatilité à court terme soit sur ces marchés parfois beaucoup plus élevée. Mais dès lors que l'on se prononce sur la base des moyennes mensuelles (ce qui est la bonne approche pour raisonner sur une longue période), il est clair que marché dérivé ou pas, on constate le même type de volatilité et I'on ne voit pas la différence de comportements des cours que les produits soient ou non dotés de marchés dérivés.

\section{Transparence et anticipation des marchés représentatifs}

Quelles sont les fonctions et les apports des marchés représentatifs aux noms desquels il convient de les défendre?

Leur premier apport est me semble-t-il la transparence. De trop nombreux marchés agricoles (physiques) rendent encore la recherche du prix de référence difficile. Le prix mondial du beurre et de la poudre de lait s'estimait encore il y a peu presque au doigt mouillé faute de bon marché de référence. Et si on doit aux travaux de Patricio Méndez del Villar le moyen d'approcher le prix mondial du riz, ce n'est toujours pas quelque chose d'évident à calculer au quotidien. Alors que d'un seul clic, chaque opérateur accède au prix du blé à Chicago, du $\mathrm{FOB}^{6}$ Rouen sur le Matif et dispose ainsi, pour en dériver les prix physiques, $d^{\prime}$ une base de référence. En second lieu, le marché est une source essentielle d'informations et d'anticipation sur la durée : il n'informe pas seulement sur la situation à un instant $\mathrm{T}$ mais permet par exemple d'intégrer, pour les céréales les caractéristiques de la fin de campagne 2008-2009, l'état des stocks, le comportement des marchés, etc. Il me semble fondamental de pouvoir disposer de cette fonction d'anticipation de long terme. Entre nous, y aurait-il eu ce colloque s'il n'y avait pas eu de marché signalant cette scandaleuse flambée des prix du printemps dernier? La fonction du marché c'est aussi de « mettre le doigt là où ça fait mal ». On peut toujours déclarer, comme cet ancien Premier ministre français également ancien ministre de l'agriculture (qui n'a pas été Président de la République, ce qui réduit le champ des possibilités) : « II faut

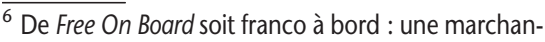
dise est achetée ou vendue FOB quand celle-ci est achetée sans les frais de transport et autres frais et taxes y afférant et sans les assurances pour cette marchandise (Wikipedia. NDLR).
}

supprimer les marchés à terme, il faut interdire Chicago "... cela serait aussi vain que penser lutter contre le froid en brisant les thermomètres. La fonction du marché en l'occurrence a peut-être été de nous rappeler de manière brutale (comme souvent réagissent les marchés) I'urgence des situations alimentaires, et que $c^{\prime}$ était là au fond le défi majeur du XXI ${ }^{\mathrm{e}}$ siècle, qui nous attend. De même, en portant le prix du pétrole à $150 \$$ le baril en juin dernier, les marchés ne nous ont-ils pas dit très simplement que le pétrole est rare, et polluant et qu'il était temps de payer le prix de cette rareté et le prix de la pollution qu'il entraîne? II serait trop facile de prétendre, comme on l'a trop souvent fait lors de la flambée des prix, que tout ceci n'est que vaine spéculation alors que les marchés ne faisaient que pointer les défis qu'il nous faut relever, et que nous avons oublié ces deux dernières décennies, en nous laissant bercer par l'illusion d'une éternelle abondance.

De même, aurions-nous tort de croire que se reproduiront chaque année les exceptionnelles conditions de la campagne 2008-2009, aujourd'hui à l'origine du repli normal que nous connaissons.

\section{Un éloge qu'il convient de limiter}

\section{Une limite morale}

Ceci étant dit, je conçois qu'il puisse y avoir quelques limites à cet éloge du marché.

La première limite qui me semble recevable et devant laquelle je $\mathrm{m}^{\prime}$ incline totalement est une limite morale. Je comprends parfaitement l'argument de ceux qui jugent défavorablement une société qui n'a trouvé que l'anticipation par le jeu des acteurs pour former le prix de l'alimentation des hommes, de ce grain symbolique par excellence associé parfois à des corpus de spiritualité les plus hauts. Et je comprends très bien ceux qui estiment choquant que l'on puisse spéculer sur le prix du blé et qui ne sont pas choqués que l'on spécule sur le prix du pétrole, du dollar, etc. Il y a une dimension symbolique, il y a une dimension morale dans l'alimentation, c'est un argument que j'accepte totalement, à partir du moment où il rentre dans un corpus idéologique, spirituel, cohérent. Simplement il ne faut pas demander au marché plus que ce qu'il ne peut donner. Dans la doctrine sociale chrétienne, héritière de Saint Thomas D'Aquin et d'Aristote il y a une notion qui est celle du juste prix que l'on retrouve aussi dans le judaïsme et dans I'Islam. Le juste prix relève de l'ordre moral, il n'appartient pas à la logique économique. Or, à un instant $\mathrm{T}$, un prix n'est juste ni injuste, ni moral ni immoral, ni même rémunérateur ni non rémunérateur.
À un moment donné, un prix constate simplement l'état de l'offre et de la demande. Sur des marchés totalement physiques il sera très imparfait puisqu'il sera déterminé sur la dernière unité de produit arrivant sur le marché, dans des situations souvent très tendues. La fonction d'anticipation apportée par les marchés représentatifs permet une réflexion plus globale et permet de restreindre les aspects marginalistes. Mais il est clair qu'on ne peut demander à un marché d'être moral ni d'être juste. La main invisible du marché est parfaite, simplement elle est a-morale et elle ne fait pas la différence entre le bon grain du petit producteur et le « mauvais » grain du très gros ou celui provenant d'un système dictatorial.

\section{Des limites fonctionnelles}

La théorie de la concurrence nous met en garde contre une deuxième limite des marchés. Pour qu'un marché soit parfait il faut que l'ensemble des acteurs ait le même degré d'intelligence des choses et puisse prendre au bon moment la bonne décision. C'est là bien entendu un pari extraordinairement optimiste sur la capacité des hommes à prendre de bonnes décisions. II suppose également une parfaite égalité des acteurs dans l'accès à l'information, dans l'accès à la prise de décision, dans l'accès aux circuits financiers leur permettant de disposer de cette fonction d'anticipation du marché. II est bien évident que c'est loin d'être partout le cas. Parce qu'ils ont accès à l'information, parce que notre "Saint Père » le Crédit Agricole peut leur permettre de financer l'achat d'options, les agriculteurs de France sont de plus en plus nombreux à utiliser les marchés. II est bien évident que ce n'est pas le cas des agriculteurs du Tiers-monde. J'ai fait autrefois partie d'une « task force » mise en place par la Banque mondiale travaillant sur la gestion des marché agricoles et où des esprits imaginatifs n'hésitaient pas à pronostiquer que les petits agriculteurs, ou même les petits groupements de producteurs de café, de cacao, de la côte africaine iraient bientôt utiliser les marchés à terme de New York. Évidemment il faut de temps en temps redescendre sur terre et se rendre compte que tout ceci n'est que vaine théorie. Si on veut aller jusqu'au bout de l'efficience des marchés, il faut accepter l'idée qu'ils ne seront efficients que si est entrepris au niveau mondial, un vaste effort d'égalisation des chances des acteurs, ce qui est loin d'être le cas aujourd'hui.

\section{De purs moments « d'exubérance irrationnelle »}

Autre limite enfin, que vient de nous rappeler l'actualité, les marchés parfois échappent à toute rationalité et connaissent de longues 
périodes $d^{\prime}$ « exubérance irrationnelle » comme le disait Greenspan dont ils se révèlent par euxmêmes incapables de sortir. C'est ce que résume I'histoire du trader qui tous les matins implorait : "Seigneur, Seigneur, je sais que ce n'est pas rationnel mais donnez-moi encore une journée de hausse et je vous assure que ce soir je dénouerai ». Jour après jour, les semaines et les mois ainsi passèrent, lorsqu'un matin le Seigneur lui répondit: «Trop tard mon fils, hier soir j'ai tout vendu ».

Cette irrationalité des marchés ne date pas d'hier. J'ai parlé de la Tulipomania au XVII e siècle aux Pays-Bas. En 1974 à Paris, la spéculation sur le sucre fit passer les prix de 2 cents à 65 cents la livre, soit à un niveau totalement délirant. Cette année, de ce point de vue, la palme a peut-être été emportée par le marché du fret maritime - un marché physique sur lequel existe aussi du dérivé, non organisé aux travers de produits que l'on appelle les FFA (Forward Freight Agreements) non régulés, des marchés très techniques. L'indice qu'utilise ce marché (le Baltic Dry Index) en prenant pour base 1000 en 1985, était à 850 en 2001, il passe à 5000 en 2004-2005, il retombe à 2000 en 2006, et atteint le 20 mai 2008, 12 000. Hier (novembre 2008) ce même indice plafonnait à 804. L'exubérante irrationalité qui a porté l'indice à cette hauteur n'était pas sans raison et se fondait par exemple sur ces files de bateaux attendant devant les ports leur chargement de charbon à destination de la Chine, avant les $\mathrm{J}$. Le problème est que cette irrationalité joue aujourd'hui dans l'autre sens et les taux du fret s'effondrent à des niveaux qui ne couvrent même plus le prix du fuel pour faire avancer les bateaux.

Les marchés peuvent effectivement connaître des moments de pure folie au cours desquels ils perdent tout contact avec la réalité physique qu'ils sont sensés représenter. Lorsque le prix du nickel passe de 5000 à 55000 dollars la tonne, pour se stabiliser aujourd'hui autour de 10000 on peut facilement imaginer les tensions qui en résultent au niveau industriel. Les marchés sont certes des modèles, de superbes machines conceptuelles parmi lesquelles les travaux de Nicole El Karaoui dont j'ai déjà cité le nom font autorité au niveau mondial. II n'en reste pas moins que les marchés, dans la mesure où ce sont des hommes qui les organisent, peuvent se révéler être le pire des systèmes. Le problème étant... que je n'en connais point d'autres à partir du moment où je suis bien obligé de constater que tous les efforts entrepris aux niveaux national et international pour les stabiliser et maintenir leur fonctionnement à un niveau de rationalité acceptable, que tous ces efforts ont échoué.

Que faire dans ces conditions? La réponse je crois peut se décliner à trois niveaux : au niveau mondial, au niveau européen et au niveau des pays du Sud.

\section{Vers une stabilisation des marchés agricoles : trois approches}

\section{Au niveau mondial}

Au niveau mondial, un certain nombre d'idées circulent selon lesquelles on pourrait organiser l'agriculture mondiale. Un mouvement ${ }^{7}$ a même été lancé en France qui disposerait de superbes modèles. Une publication de l'Ifpri ${ }^{8}$ (Physical and virtual global food reserves to protect the poor and prevent market failures ${ }^{9}$ ) de Joachim von Braun and Maximo Torero, plaide pour la mise en place d'une sorte de structure mondiale qui pourrait intervenir directement afin de limiter les effets de la spéculation sur les marchés organisés. Toutes ces propositions je l'avoue, quoique généreuses, me laissent sceptique. Je fais partie de la génération de ceux qui furent bercés par la résolution 93/IV de la Cnuced $^{10}$ de Nairobi en 1976 qui adopta le programme intégré pour les produits de base.

Les premiers accords internationaux sur la stabilisation des marchés de matières premières agricoles étaient même antérieurs et remontaient en fait au lendemain de la crise de 1929. C'est dans le cadre de la conférence économique de Londres de juin-juillet 1933 (qui fut un échec à partir du moment où le gouvernement de Roosevelt dévalua le dollar) que furent négociés deux accords internationaux de stabilisation, l'un sur le blé, l'autre sur le sucre. Et depuis cette date c'est à Londres que siègent le Conseil international du blé (devenu le $\mathrm{CIG}$ ), et l'Organisation internationale du sucre.

Après-guerre, de nouveaux accords furent adoptés, sur le blé, le sucre, le café. La dynamique de Nairobi (1976) - où fut mis en place le Fonds commun pour les produits de base - permit la signature de nouveaux accords sur le cacao, sur le caoutchouc naturel, etc. Mais force est de constater qu'aucun de ces accords n'a jamais véritablement fonctionné. Or le monde était à l'époque plus « facile » dans la mesure où les monnaies étaient stables et où la plupart des grandes matières premières industrielles et énergétiques connaissaient elles aussi une grande stabilité. Benoîtement, une stabili-

\footnotetext{
7 [Momagri.NDLR]

8 International Food Policy Research Institute

${ }^{9}$ www.ifpri.org/pubs/bp/bp004.pdf.

${ }^{10}$ Conférence des Nations unies sur le commerce et le développement
}

sation du prix du blé, dans ces conditions, nous paraissait réalisable. Nous n'y sommes jamais parvenu. II faut écouter ce que nous apprend I'histoire. Un mouvement pour l'agriculture mondiale est une idée certes généreuse mais malheureusement non réaliste. On évoque volontiers aujourd'hui un second Bretton Woods pour la surveillance des marchés financiers, mais personne ne parle de restabiliser les marchés monétaires (ou bien se serait la création d'une monnaie unique et nous n'en sommes pas encore là). Les échecs du passé devraient nous éclairer. Le seul accord qui ait jamais fonctionné est celui sur le caoutchouc naturel et ceci pour une raison très simple : il s'apparentait un peu à un cartel dans la mesure où $90 \%$ de l'offre provenaient de trois pays voisins des pays (la Malaisie, I'Indonésie et la Thaillande) et étaient souvent contrôlés par les mêmes familles chinoises. Ce qui ne les a pas empêchés, à un moment donné, de saborder l'organisation internationale du caoutchouc qui fonctionnait avec un stock régulateur plutôt que de continuer à en payer les coûts. Car la stabilisation coûte chère. Si bien qu'il ne me semble ni possible ni même souhaitable (d'un point de vue du rapport coût/efficacité) de chercher à faire quoi que ce soit à l'échelle internationale. Mis à part l'idée portant sur l'organisation d'un stockage de précaution pour les interventions d'urgence dans le cadre du Programme alimentaire mondial, je pense et je demeure persuadé qu'il vaut mieux se placer, au niveau international, sous la logique des marchés.

\section{Au niveau européen}

Au niveau européen, pour moi, il faut entériner ce que la Commission a décidé, il faut libéraliser notamment le marché des céréales. Et, de ce point de vue, mettre fin à l'arbitraire des fonctionnaires bruxellois. Aujourd'hui dans le fonctionnement de la logique de Bruxelles, je préfère encore que ce soit les marchés qui spéculent que des fonctionnaires de la Commission qui soient amenés à dire : « je donne de la restitution ou je ne la donne pas », ce qui est un acte purement spéculatif.

Il est vrai que c'est un changement profond. Je fais partie de ceux qui sont partisans de la fin des prix d'intervention, de la fin de toutes les régulations sur le marché céréalier européen. Et je pense, même si cela va à l'encontre de tout ce qui est proclamé le plus souvent au niveau syndical, y compris dans cette maison (I'ACPA), que nous nous en porterons tous bien mieux.

\section{Les pays du Sud}

Pour les pays du Sud, mon raisonnement est totalement différent et je n'hésite pas à affirmer 
haut et fort qu'il est hors de question de leur demander d'appliquer les mêmes recommandations. La plupart des pays africain du Sud ne sont pas mûrs pour quelque marché que ce soit et il me semble totalement légitime (et Pascal Lamy en est $d^{\prime}$ accord) que les PMA ${ }^{11}$ adoptent et maintiennent des politiques agricoles conçues sur le modèle de la PAC, leur permettant d'instaurer une garantie des prix. Le problème est qu'une telle politique, on le sait, ne peut être financée que de deux manières : par le consommateur ou par le contribuable. Or au sein des PMA, le contribuable est absent et le pouvoir d'achat du consommateur urbain à peu près inexistant. C'est ici que l'aide internationale serait la plus utile. J'ai vu avec grand intérêt les initiatives récentes des fondations Gates et Buffett qui, dans un certain nombre de pays d'Afrique de l'Est, commencent à finan- cer des systèmes de garantie de prix aux producteurs. Incontestablement, si nous voulons aller dans le sens de cette idée à laquelle j'adhère totalement, celle du « Droit des peuples à se nourrir eux-mêmes ${ }^{12}$ ", il nous faut accepter de les aider à garantir des prix rémunérateurs. C'est pour moi une évidence, même si cela sera très difficile puisque faute d'être assez fort sur un plan économique il leur faudra compter sur l'aide internationale.

Pensons ici à I'Inde, qui a su maintenir envers et contre tous une politique agricole - et Dieu sait si nous l'avons brocardée, accusée de contribuer à nourrir plus les rats que les Indiens. L'Inde disposait il est vrai de la puissance politique et économique pour le faire alors que nombre de pays du Tiers-monde, faute des mêmes armes, cédèrent à la facilité en recourant aux importations (sources également d'occultes et appréciables commissions). Et que constate-t-on aujourd'hui ? II y a bien sûr en Inde des gens qui souffrent toujours de malnutrition et de pauvreté mais l'Inde n'a pas connu d'émeutes de la faim et demeure pour moi un modèle certain.

Pour conclure, je dirais qu'avec la crise que nous vivons, celle des matières premières agricoles, j'ai bien conscience notamment en France que le marché est devenu le bouc émissaire parfait, source de tous les maux à cause duquel il faudrait en revenir à des prix rémunérateurs, restabiliser, etc.

J'en suis désolé mais le marché ne peut pas être chargé de tous les maux : au contraire il me semble avoir très bien joué son rôle, je n'en dirais pas autant de nombre de nos instances politiques.

\footnotetext{
11 Pays les moins avancés

12 titre du livre de Bertrand Hervieu
} 